\title{
Bloqueo infraclavicular guiado por ultrasonido como técnica anestésica quirúrgica para cirugía de extremidad superior, experiencia en un hospital universitario
}

\author{
Sebastián layera', Julián Aliste ${ }^{1}$ Daniela Bravo', Diego Fernández¹, Alvaro Jara
}

\begin{abstract}
Introducción: El bloqueo infraclavicular (BIC) guiado por ultrasonido es una técnica poco utilizada en nuestro medio para anestesia quirúrgica. La ejecución del BIC mediante una inyección única dorsal a la arteria axilar buscando el signo de la doble burbuja[1] ha demostrado una alta efectividad en estudios clínicos randomizados recientes.

Objetivo: Describir la experiencia del uso del BIC inyección única como anestesia quirúrgica para cirugía de extremidad superior, con énfasis en la dinámica del bloqueo, su efectividad como técnica anestésica, así como también su incidencia de complicaciones relacionadas al procedimiento.
\end{abstract}

Material y Métodos: Previa autorización del comité de ética científica se obtuvo información preliminar de un estudio clínico randomizado en curso, en el cual se reclutaron 150 pacientes sometidos a cirugía de extremidad superior bajo anestesia regional. Se realizó un BIC guiado por ultrasonido con Lidocaína $1 \%$ - Bupivacaína 0,25\% - epinefrina $5 \mu \mathrm{g} / \mathrm{mL}$ con un volumen de $35 \mathrm{ml}$. Durante la ejecución del bloqueo se registraron el tiempo de ejecución, número de pases de aguja, dolor procedimental y complicaciones. Posteriormente, un evaluador ciego registró la tasa de éxito del bloqueo, la latencia y la incidencia de anestesia quirúrgica. Se realizó también un control telefónico a los 7 días en busca de complicaciones neurológicas.
Resultados: Desde julio de 2017 hasta mayo de 2018 se incluyeron 150 pacientes. La tasa de éxito del BIC guiado por ultrasonido fue de $94,6 \%$, con una incidencia de anestesia quirúrgica de un $96 \%$. Se obtuvo un tiempo de latencia de $19,7(5,6)$ minutos. El tiempo promedio de ejecución fue de 6,4 $(2,2)$ minutos, mientras la mediana del número de pases de aguja y el dolor procedimental fueron 2 y 1 respectivamente. Las complicaciones durante el procedimiento registradas fueron parestesias en $6(4 \%)$ pacientes y punción vascular en 7 (4,6\%). En el control a 7 días sólo un paciente presentó déficit neurológico (parestesias) con recuperación completa antes de las 2 semanas posteriores al bloqueo.

Conclusiones: EI BIC guiado por ultrasonido es una técnica altamente efectiva como anestesia quirúrgica para cirugía de extremidad superior en nuestra experiencia, siendo esta última concordante con reportes recientes de otros centros universitarios. Aunque el tiempo de latencia registrado puede ser mayor comparado con otros abordajes, su simplicidad favorece una ejecución rápida con menos pases de agujas y escaso dolor relacionado con el procedimiento. Las complicaciones observadas durante y posterior al bloqueo están dentro del rango reportado en la literatura y sin mayor repercusión clínica.

Hospital Clínico de la Universidad de Chile. Chile. 